\title{
1 Simple models highlight differences in the walking biomechanics of \\ 2 young children and adults
}

3 Vivian L. Rose and Christopher J. Arellano*

4

5 Department of Health and Human Performance,

6 University of Houston, Houston, TX 77024 USA

7

8 *Corresponding Author:

9 Christopher J. Arellano

10 Department of Health and Human Performance

11 University of Houston

12 Houston, TX 77204

13 Tel. 713-743-9840

14 carellano@uh.edu

15

16

Key words (3-6): child, locomotion, energetics, metabolic, energy 


\section{Abstract (250 max)}

Adults conserve metabolic energy during walking by minimizing the step-to-step transition work performed by the legs during double support and by utilizing spring-like mechanisms in their legs, but little is known as to whether children utilize these same mechanisms. To gain a better understanding, we studied how children (5-6 years) and adults modulate the mechanical and metabolic demands of walking at their preferred speed, across slow

$23(75 \%)$, preferred (100\%), and fast (125\%) step frequencies. We quantified the 1) positive mass-

24 specific work done by the trailing leg during step-to-step transitions and 2) the leg's spring-like

25 behavior during single support. On average, children walked with a $36 \%$ greater net cost of 26 transport (COT; $\mathrm{J} / \mathrm{kg} / \mathrm{m})$ than adults $(p=0.03)$, yet both groups increased their net COT at varying 27 step frequencies. After scaling for speed, children generated $~ 2$-fold less trailing limb positive scaled mechanical work during the step-to-step transition $(p=0.02)$. Unlike adults, children did not modulate their trailing limb positive work to meet the demands of walking at $75 \%$ and $125 \%$ of their preferred step frequency. In single support, young children operated their stance limb with much greater compliance than adults $(\hat{k}=6.23$ vs. $11.35 ; p=.023)$. Our observations suggest

32 that the mechanics of walking in children 5-6 years are fundamentally distinct from the mechanics of walking in adults and may help to explain a child's higher net COT. These insights

34 have implications for the design of assistive devices for children and suggest that children cannot 35 be simply treated as scaled down versions of adults. 


\section{Introduction}

Historically, simple models have been instrumental for understanding how humans control the lifting and forward motion of the body's center of mass during walking. Simple models reduce the body to a point mass supported by two stiff or, alternatively, spring-like struts that characterize an "optimal" transfer or redirection of mechanical energy of the center of mass (Antoniak et al., 2019; Cavagna, GA and Margaria, R, 1966; Donelan et al., 2002; Geyer et al., 2006). With more efficient transfer or redirection, less mechanical work is required of the muscles and tendons to propel the center of mass. Lowering the mechanical work required for walking is associated with lowering the demand for consuming metabolic energy. This is now recognized as a fundamental principle of locomotion biomechanics, and one might reason that these simple models, for which these principles were derived from experimental data on adults, could be applied to young children.

However, recent insights suggest that minimizing the work performed by the legs is a dynamic process that must be learned, and which may depend on the biological and morphological constraints of the body. Bril et al. (2015) use a simple model to show how young, typically developing children (1 to 5 years) gradually learn to modulate the distance between their center of mass and center of pressure, which dynamically changes during the gait cycle. Modulating the distance between the center of mass and center of pressure is governed by the forces that the legs generate in the vertical and anterior-posterior directions. But in particular, it appears that generating forces in the anterior-poster direction (i.e. propulsive forces) is a skill learned much later in childhood, which requires tuning and control to achieve balance and elicit the desired motion in the forward direction (Brenière and Bril, 1998; Bril et al., 2015). Several studies have also highlighted differences between the typical ground reaction profiles generated by young children and adults (Dewolf et al., 2020; Takegami, et al., 1992), and have used simple models to suggest mechanical work minimization may not be the optimal strategy for young children (Usherwood et al., 2018b). This raises the question as to how the walking pattern of young children should be modeled, recognizing that that their walking mechanics may not simply be scaled down versions of adults.

For the same simple models to apply and scale to children, several assumptions must be 
forces characteristic of walking will scale in proportion so that comparisons can be made across "scaled speed." Dimensionless speed, e.g. the Froude number, is a widely used method to scale when comparing walking dynamics between children and adults; however, the Froude equation is based on the idea of dynamic similarity (R.M. Alexander and A.S. Jayes, 1983). Dynamic similarity means that multiplying all linear dimensions, time intervals, and forces by constant factors would result in identical walking patterns. Deviations from dynamic similarity are observed in young children (Kramer and Sarton-Miller, 2008; Usherwood et al., 2018b), and the Froude number makes no allowance for differences in shape. Differences in shape, or anthropomorphic proportions, may be an important consideration when making metabolic comparisons within a species, or in animals relatively close in size (Kramer and Sylvester, 2013). Alternative scaled speeds, like the Strouhal and Groucho (Alexander, 1989; Blickhan, 1989; McMahon et al., 1985) may be used when elastic forces are considered, such as in running or when a spring-like element is added to a walking template (Geyer et al., 2006).

Second, we must assume that young children have the muscular capacity to generate and control the force and mechanical work required to move the center of mass in the most economical way possible. During walking, the muscular capacity of muscles surrounding the hip and the ankle joints are particularly important for propulsion and stability (Brenière and Bril, 1998; Ishikawa et al., 2005). Compared to adults, young children display many differences in muscle and tendon properties. They have a proportionally smaller cross-sectional area of plantar flexor muscles, differences in Achilles tendon compliance (Waugh et al., 2012), slower rates of muscle force development, and lower magnitudes of relative maximum force production during isolated contractions (Radnor et al., 2018). Further experiments would help to determine how these properties influence functional or metabolic differences during walking. However, given the importance of muscles and tendons to supporting body weight and redirecting the center of mass - both critical for economic walking in adults (Donelan et al., 2002; Grabowski et al., 2005) - it is conceivable that immature muscle-tendon capacities influence a young child's "optimal" walking solution. Of the few studies that specifically examine center of mass motion in children, normalized center of mass amplitudes in the sagittal plane are proportionally greater

94 than in adults until around the age of 7 to 9 years (Dierick et al., 2004), which may reflect a 
Finally, we must assume that children minimize their metabolic cost during walking, and that this cost is proportional to their size. Overall, when examining walking at a range of speeds, children over 6 years exhibit an "optimal” speed that minimizes cost (DeJaeger et al., 2001). Yet, surprisingly, at "optimal" speed, the net mass-specific cost of transport (COT, J/kg/m) is up to 33\% higher in children less than 9 years old (Bolster et al., 2017; DeJaeger et al., 2001; Morgan et al., 2002). With walking speeds normalized to the Froude number, DeJaeger at al. found that differences in net COT may largely be reduced, thus body size alone may account for the higher net COT observed in young children (DeJaeger et al., 2001). However, Schepens et al. found that efficiency, defined as the ratio of the total mechanical power to the net energy consumption rate, is much lower in younger children (Schepens, 2004). If mechanical power incurs a relatively greater cost in younger children, this again raises the question as to the extent to which muscular capacity and control plays a role in explaining higher net COT. Taken together, these insights suggest a functional, mechanistic explanation for why young children are less economical at walking than adults (Schepens, 2004).

To gain greater insight into a potential explanation, our objective was to use the methodology of simple biomechanical templates to investigate how the generation of limb forces and the resulting motion of the center of mass in children may differ from adults, and thus may relate to their metabolic differences. We took a systematic approach by breaking down the walking gait cycle into distinct phases of double and single support. In mature walking patterns, the legs must redirect the center of mass from a downward and forward velocity to an upward and forward velocity during double support, when both the leading and trailing limb are in contact with the ground (Kuo et al., 2005). This redirection, also called the step-to-step transition, is considered a major determinant of the metabolic cost of walking in adults (Donelan et al., 2002), and has also been studied in 12-18 month old toddlers (Hallemans et al., 2004). For our first aim, we sought to compare the positive external mechanical work $\left(\mathrm{W}^{+}{ }_{\text {ext }}\right)$ performed by the trailing leg in young children (age 5-6 years) and adults (age 18-30), with adults representing the "ideal" behaviour. We hypothesized that 1) after accounting for differences in mass and dimensionless walking speed, the trailing limb in young children would produce less $\mathrm{W}^{+}$ext during double support. This hypothesis was based on literature that supports the idea that during development, children gradually tune and increase their anterior-posterior propulsive forces (Bril et al., 2015), indicating that when normalized to body mass, children aged 3-8 years generate less 
128

129

130

131

132

133

134

135

136

137

138

139

140

141

142

143

144

145

146

147

148

149

150

151

152

153

154

155

156

157

power at the ankle than adults (Chester et al., 2006). Young children also typically produce asymmetric vertical ground reaction forces while walking (Preis et al., 1997; Takegami, M.D., 1992; Usherwood et al., 2018a), suggesting that children do not transition from one step to the next like adults.

Inspired by the bipedal walking spring mass model of Geyer et al (2006), we also set out to compare how young children and adults modulate the distance between their center of mass and center of pressure during single support, which reflects the spring-like behavior of the leg. From the perspective of a spring-mass model (Geyer et al. 2006), the redirection of the center of mass can be achieved by the release of elastic energy that was stored during single support. At the beginning of single support, when the leg extends and center of mass rises, elastic energy that was absorbed during the double support may be released, and subsequently, as the center of mass descends during the second half of single support, elastic energy can be stored in preparation for double support (Donelan et al., 2002). This appears to be the ideal behavior in adults, whereby step-to-step transitions are facilitated by the ability of the leg to store and release elastic energy and thus, operate much like a spring. In contrast to adults, a child's leg consists of immature muscles and tendons and may not operate like an ideal spring. In addition, single support is also the phase that requires postural adjustment to stabilize the body in an upright position. The center of mass is accelerated forward and sideways at the same time toward the swing leg (Bril et al., 2015), having a destabilizing effect. Modulating the distance between the center of mass and center of pressure, i.e. the spring length, during single support may be less precise in children and require more muscle cocontraction and activation (Grosset et al., 2008; Lambertz et al., 2003). These biomechanical constraints would ultimately raise the net COT during walking and therefore, we hypothesized that 2) after considering dimensionless speed and scaling for size, the stiffness of the leg spring $(k)$ in children would differ from adults.

For both of our main hypotheses, we also explored whether young children would modulate their trailing limb work and spring stiffness in the same way as adults when meeting the demands of walking at a fixed speed, but at step frequencies slower and faster than preferred. Therefore, we tested these hypotheses under conditions in which children and adults walked at their preferred speed and across a range of slow to fast step frequencies set at $75 \%, 100 \%$, and $125 \%$ of their preferred step frequency. Faster step frequencies involve shorter step lengths, 
158

159

160

161

162

163

164

165

166

167

168

169

170

171

172

173

174

175

176

177

178

179

180

181

182

183

184

185

186

while slower step frequencies involve longer step lengths. At slower step frequencies, the angle between the legs at the instance of double support increases and vice versa at faster step frequencies. According to the individual limbs model, positive mechanical work at push-off depends on both the center of mass velocity and on the angle between the legs (Donelan et al., 2002). Following that model, we expected more propulsive trailing limb work at the slower step frequency condition for both adults and children. In regards to the the bipedal spring mass model, we expected that a slower step frequency would yield a decrease in the touchdown angle of the leading limb; however, both touchdown angle and $k$ vary within a large range (Geyer et al., 2006), so we could not formulate a predictive hypothesis as to how $k$ might change with step frequency.

Overall, we reasoned that changes in the mechanical demands of modulating step frequency would result in greater metabolic demands for young children. Specifically, when challenged to walk at the slowest step frequency, which requires the largest step lengths, young children would likely consume greater rates of metabolic energy, and hence a greater net COT when compared with the other step frequency conditions. Given known differences in a child's muscle and tendon morphology, ankle power generation, and lower walking efficiencies, we hypothesized that when compared to adults, 3) young children would incur a greater net COT to modulate their step frequency than adults. In particular, we expected that the legs would be required to generate the greatest amount of mechanical work at the slowest step frequency condition, and that the extra mechanical demand would be more costly for children than for adults.

\section{Materials and Methods}

\section{Experimental Procedures}

Healthy young adults (aged 18-32 years, $n=8$ ) and typically developing, healthy children (aged 5-6 years, $n=8$ ) were recruited locally. Adult subjects gave their written informed consent to participate. Parents of child subjects gave permission and written informed consent, and 
children gave verbal assent to participate, in accordance with ethical guidelines and approved by University of Houston Institutional Review Board. To ensure that we captured the most natural walking patterns and comfort with the testing environment, children visited the lab for a preliminary 1.5 -hour practice session the previous day. For the child cohort, a parent was present during all parts of the experiment and gave encouragement when necessary.

During the day of the experiment, subjects arrived having fasted and refrained from caffeine or exercise for at least 3 hours. Upon arrival to the lab, subjects rested for 10 min before we measured their standing metabolic rate for 5 minutes using an open circuit TrueOne 2400 metabolic system (ParvoMedics, Inc. Sandy, UT USA). The metabolic system was calibrated immediately before each session using standard gases and a $3 \mathrm{~L}$ syringe. Following the standing trial, reflective markers $(15.9 \mathrm{~mm})$ were placed according to manufacturer guidelines (Lower Body Plug-in Gait, 100 Hz; Vicon 12-camera system, Nexus 1.8.5, Vicon, Oxford, UK) and the standard scaling and calibration protocol of the Nexus 1.8.5 software was followed. An additional marker was placed at the subject-specific location of the center of mass as determined by the reaction board method (Enoka, 2015). All subjects (Table 1) wore their own shirt, bike shorts, and tennis shoes with a heel-sole difference no greater than $6.4 \mathrm{~mm}$.

Each subject then walked on a level dual belt instrumented treadmill $(1000 \mathrm{~Hz}$; Bertec Co. Columbus, OH USA) at their preferred speed. Preferred speed was obtained through feedback by having subjects walk at increasing increments of speed (starting from $0.3 \mathrm{~m} / \mathrm{s}$ in the child group and $0.5 \mathrm{~m} / \mathrm{s}$ in the adult group). Once they reached a preferred walking speed, speed was increased again and lowered if necessary, to confirm their preferred walking speed (Arellano et al., 2009). Following a 5 min rest, subjects then walked for 5 minutes at preferred walking speed and were instructed to match their step frequency to the sound of a metronome at 75, 100, and $125 \%$ of preferred step frequency (order randomized). Subjects sat for a 5 min rest period between trials. All subjects achieved a steady rate of metabolic energy consumption with respiratory exchange ratios (RER) remaining within the normal physiological range below 1.0.

\section{Data analysis}

Walking metabolic power $\left(\mathrm{W} / \mathrm{kg}\right.$ ) was calculated from average $\dot{V} \mathrm{O}_{2}$ and $\dot{V} \mathrm{CO}_{2}$ during the last 3 minutes of each trial (Brockway, 1987). The average quiet standing value was subtracted from the average walking value to yield net metabolic power $(\mathrm{W} / \mathrm{kg})$. Net metabolic power was 
217

218

219

220

221

222

223

224

225

226

227

228

229

230

231

232

233

234

235

236

237

238

239

240

241

242

243

divided by speed $(\mathrm{m} / \mathrm{s})$ to obtain net COT expressed in units of $\mathrm{J} / \mathrm{kg} / \mathrm{m}$. Data were filtered using a $4^{\text {th }}$ order, zero-lag low-pass Butterworth filter with a cut-off frequency of $15 \mathrm{~Hz}$ for force and $6 \mathrm{~Hz}$ for kinematics. All data were processed in Matlab (R2018b, The MathWorks, Massachusetts, USA) and custom code was written to calculate values for limb work and power (Donelan et al., 2002) and for values of leg stiffness, $k$, following a spring-mass model (Geyer et al., 2006). To identify gait cycle events and periods of double and single support, we defined touchdown and toe-off as the instant when the vertical ground reaction force (GRF) crossed a threshold of 5\% body weight. Double support was then defined as the portion of the gait cycle after touchdown of the leading limb, and before toe-off of the trailing limb. Single support was defined as the portion of the gait cycle between toe-off and touchdown when only one foot was in contact with the ground.

Individual Limbs Method. Starting from the 3 min mark of each 5 min walking trial, filtered force data from periods of double support were aggregated to determine lead leg and trail leg power and work. Following the method of Donelan et al., the velocity of the center of mass was determined by single integration. Then the external mechanical power generated by the trailing and leading limb (as shown in Fig. 2) was determined by summing together the dot product of the force and velocity of the center of mass acting in each direction (Donelan et al., 2002).

$$
\text { (1a) } P_{\text {lead }}=\text { Force }_{x, \text { lead }} \cdot \text { velocity }_{x, \text { com }}+\text { Force }_{y, \text { lead }} \cdot \text { velocity }_{y, \text { com }}+\text { Force }_{z, \text { lead }} \text {. }
$$

$$
\text { velocity }_{z, \text { com }}
$$

$$
\text { (1b) } P_{\text {trail }}=\text { Force }_{x, \text { trail }} \cdot \text { velocity }_{x, \text { com }}+\text { Force }_{y, \text { trial }} \cdot \text { velocity }_{y, \text { com }}+\text { Force }_{z, \text { trail }} \text {. }
$$

$$
\text { velocity }_{z, c o m}
$$

The magnitude of positive mechanical work of the trailing limb $\left(\mathrm{W}_{\text {trail }}^{+}\right)$, expressed in Joules, was then calculated as the time integral of the positive portions of trailing limb power from equation (1b). For comparisons of young children and adults at their preferred speed $(\mathrm{m} / \mathrm{s})$, the total $\mathrm{W}^{+}$trail was normalized to mass (Schepens, 2004) and averaged across the first 100 double supports starting from the 3 min mark. 
Single Support Spring Mass Method. Again, starting from the 3 min mark of each 5 min walking trial, filtered force data from periods of single support were aggregated. We first found the position of the center of mass using double integration and then defined a vector from the center of mass to the foot's centre of pressure to represent a virtual 2D spring in the sagittal plane. The markers at the center of mass and pelvis were used as an offset to reflect the absolute position of the center of mass after double integration, such that the center of mass was assumed to be within the body and half the distance between the anterior superior iliac spine and posterior superior iliac spine markers. Following the work of Gard et al. (2004), we assume that this method is a close approximation to the exact location of the center of mass and more importantly, should reflect the trajectory and amplitudes that the center of mass undergoes during each step. Since we were primarily interested in the lift and propulsion of the center of mass, we reasoned that a sagittal spring was an adequate starting point for comparisons. Plotting resultant 2D GRF (N) values as a function of spring length $(\mathrm{m})$ allowed us to calculate the slope of the best fit line via a least-squares regression analysis (Fig. 3). The mean of the absolute slopes estimated from the first 30 steps was reported as the value of $k$. This decision was based on our ranging from 0.5 to 0.9 . On the other hand, children exhibited spring-like behavior that was much less consistent, so for fair comparisons, we limited the $\mathrm{R}^{2}$ values to 0.5 which allowed us to aggregate a minimum of 30 steps.

\section{FIGURE 3 ABOUT HERE} superior iliac spine to the distal tip of the medial malleolus. The dynamic walking model equations only apply when the body conforms to inverted pendulum dynamics (Kuo et al., 2005), so we chose to consider Froude speed in our comparison of individual limb work.

$$
\text { (2) } F r=\frac{v^{2}}{g L_{l e g}}
$$

275 To normalize $k$, we used the Groucho speed, which was originally developed as a vertical speed parameter, combining interactions between effective vertical spring stiffness, gravity, impact 
277 velocity, and body mass (McMahon et al., 1985). It has been mathematically adapted and used in

2782 dimensions by also considering leg length (Blickhan, 1989). Essentially, for both the fore-aft

279 and vertical directions, the Groucho speed can be calculated using equation ( $3 a$ ) and normalized

280 to leg length, where $v$ is the resultant 2D velocity at the instant of touchdown, $g$ is gravity, and $\omega$

281 is the natural frequency of the system as determined by spring stiffness, $k$, and body mass, $m$, as

282 in equation $(3 b)$.

Because the Groucho number is a dimensionless speed based on a spring-mass model, we planned to use Groucho speed as a covariate in our comparison of $k$, but as explained in the Statistical analysis below, we instead used $\hat{k}$, which represents its non-dimensional form. Touchdown angle was determined in the sagittal plane as the angle formed at touchdown between a line created by the center of mass and lateral malleolus marker with respect to the horizontal. We aggregated the touchdown angles from the entire 3 minutes and report the means

291 in Table 2.

We used separate mixed R-ANCOVA's with a priori planned comparisons to test for differences in $\mathrm{W}^{+}$trail, non-dimensional spring stiffness $(\hat{k})$, touchdown angle, and net COT. After checking for normality, we compared differences between groups at the 3 step frequencies by defining age and step frequency as a between and within subjects fixed factor, respectively. To account for differences in size and speed between children and young adults, we included in our analysis of $\mathrm{W}^{+}$trail, the dimensionless speed Froude as a covariate.

Upon initial inspection of our Groucho speeds, we removed an outlier in the adult group so statistical comparisons for $k$ were based on $n=7$ in the adult group. Based on independent $t$-test at each step frequency (Table 2), we did not detect significant differences in Groucho speed

303 between groups and therefore did not require Groucho as a covariate. We did not use Froude as a covariate for this analysis, since we were only concerned with single support and were using a theoretical model that is based on spring-mass dynamics, not pendular dynamics. To account for 
306

307

308

309

310

311

312

313

314

315

316

317

318

319

320

321

322

323

324

325

326

327

328

329

330

331

332

333

334

differences due to body size in our comparison of $k$, we transformed to non-dimensional $\hat{k}$ using equation 4 , where $l$ is the max spring length, $m$ is body mass, and $g$ is gravity.

(4) $\hat{k}=\frac{k l}{m g}$

For comparisons of touchdown angle and net COT $(\mathrm{J} / \mathrm{kg} / \mathrm{m})$, we included Froude as a covariate because these variables strongly depend on walking speed. During post-processing, we discovered that a hardware malfunction caused the force data to be erased for one child subject walking at $125 \%$ of their preferred step frequency. Therefore, statistical comparisons for massnormalized $\mathrm{W}^{+}$trail and $\hat{k}$ were based on a sample size of $n=7$ in the child group. For our planned comparisons, we used independent $t$-tests when normality was met or Mann-Whitney U tests when normality was not met. Finally, we plotted mass-normalized $\mathrm{W}^{+}$trail, $\hat{k}$, and net COT across conditions for both groups. All data was analysed in SPSS with statistical significance set at 0.05 (version 26, IBM, Armonk, NY).

\section{Results}

Touchdown angle. We used Froude as a covariate to compare touchdown angle between groups and confirmed that touchdown angle, an indirect gauge for detecting differences in step length, was changing with step frequency as expected. A significant main effect for step frequency $\left(F_{2,24}=3.324, p=.027, \eta^{2}=.217\right)$ revealed that touchdown angle increased on average from $\sim 72$ to 78 degrees when walking from the relatively slow to fast step frequencies (Table 2).

We did not detect a main effect for group or an interaction effect between group and step frequency, indicating that in response to the changing step frequencies, children and adults altered their touchdown angle in the same manner.

Double Support Mechanics. Prior to conducting the statistical analysis, data were inspected to ensure assumptions were met for a repeated measures-ANCOVA, with Froude speed as an appropriate covariate. We detected a significant within-subjects difference of $\mathrm{W}^{+}$trail $/ \mathrm{kg}$ across step frequencies $\left(F_{2,24}=3.736, p=.039, \eta^{2}=.237\right)$. As expected in adults, overall positive propulsive work was greater at longer step lengths (75\% preferred step frequency) and less at shorter step lengths (125\% preferred step frequency; Figure 4A). This was not so in the child group, as confirmed by our detection of an interaction effect $\left(F_{2,24}=5.216, p=.013, \eta^{2}=.303\right)$, 
335

336

337

338

339

340

341

342

343

344

345

346

347

348

349

350

351

352

353

354

355

356

357

358

359

360

indicating that children did not modulate the amount of $\mathrm{W}^{+}$trail $/ \mathrm{kg}$ when walking at longer and shorter steps associated with the $75 \%$ and $125 \%$ step frequency condition, respectively (Figure 4A). Between group comparisons across step frequency conditions confirmed that after accounting for differences in Froude speed, children and adults generated $0.06 \mathrm{~J} / \mathrm{kg}$ and $0.12 \mathrm{~J} / \mathrm{kg}$ of trailing limb mass-specific positive work, respectively $\left(F_{1,12}=14.106, p=.002, \eta^{2}=.54\right)$. Preplanned ANCOVA comparisons between groups confirmed that children generated less $\mathrm{W}^{+}{ }_{\text {trail }} / \mathrm{kg}$ than adults at each step frequency condition (Table 2 , all $p<.05)$.

Single Support Spring Mass Mechanics. After checking that the statistical assumptions were met for a repeated measures ANOVA, we detected a main effect for group $\left(F_{1,12}=6.831, p<.001\right.$, $\left.\eta^{2}=.363\right)$ and step frequency $\left(F_{2,24}=46.43, p<.001, \eta^{2}=.795\right)$, but no interaction effect $(p=0.41)$. At the slow step frequency associated with longer steps, $\hat{k}$ decreased, and at the fast step frequency associated with shorter steps, $\hat{k}$ increased (Fig. 4B). Overall, children walked with an average $\hat{k}$ equal to 6.82 , almost 2 -fold more compliant than adults, who walked with an average $\hat{k}$ equal to 11.35 . The much more compliant $\hat{k}$ observed in children was consistent at each step frequency condition (all $p$ 's $<.05$ for all a priori independent $t$-tests; Table 2).

Net Metabolic Cost of Transport. Prior to conducting the analysis, data were inspected to ensure assumptions were met for a mixed design and that the Froude number was an appropriate covariate. A main effect for step frequency $\left(F_{2,28}=19.656, p<.001, \eta^{2}=.584\right)$ revealed a higher net COT when walking at step frequencies above and below preferred. We also detected a main effect for group $\left(F_{1,14}=31.84, p<.001, \eta^{2}=.695\right)$, revealing that the net COT was on average, $36 \%$ higher in children (Fig. 4C). However, we did not detect an interaction effect $(p<0.05)$, indicating that across slow and fast step frequencies, the net COT changed in a similar way for adults and children. And finally, when adjusted for group differences in the Froude number, the mean values show that in both children and adults, the net COT increased when walking at the relatively slow and fast step frequencies.

FIGURE 4 ABOUT HERE

\section{Discussion}


We analyzed experimental data using simple models of walking to compare center of mass mechanics and net COT in a group of young children to those of adults, who represent the ideal behavior these simple models are based upon. One key finding was that trailing limb positive work, $\mathrm{W}^{+}{ }_{\text {trail }} / \mathrm{kg}$, was significantly lower across conditions in children. This supported our first hypothesis. However, in contrast to the other variables tested, the magnitude of this difference changed depending on the step frequency condition. The largest deficit was evident at the $75 \%$ step frequency-the condition that required subjects to take the longest steps. From Donelan et al., (2002) and Kuo et al. (2005), it is predicted that longer steps will increase the collision of the lead leg with the ground, effectively increasing negative work generated by the leading limb. To account for this greater negative work, the trailing limb must generate more positive work. Yet children did not generate the positive work that would be expected to account for the increased collision forces that are associated with taking long steps at a fixed speed.

When walking at the preferred step frequency condition, we found that on average, the trailing limb in children is used to both absorb and generate work, while in adults, the trailing limb only generates positive work, providing almost $100 \%$ of all their propulsive positive work (e.g., see Fig. 2, middle column). In adults, there seems to be a clear role for the trailing limb to generate positive work and the leading limb to generate negative work, whereas young children used both limbs to generate both positive and negative work. Halleman's et al. (2004) reports that in toddlers (12-18 months), an inefficient inverted pendular mechanism of energy exchange contributes to differences in external mechanical work. Toddlers are described as utilizing a "tossing gait" where work that is performed to lift the center of mass against gravity is much greater than work that is performed to propel the center of mass. In our age group of 5-6 years, gait patterns are considered more mature than in toddlers. Yet, when partitioning the positive and negative work generated by each limb double support, we also found (as in Hallemans et al.) that our child subjects tended to generate more mass-specific work to lift the center of mass than in adults. However, one key difference was that the mass-specific work to lift versus to propel the center of mass was approximately equal for our child subjects (Fig. 5), suggesting a possible shift with age toward more mature patterns when work to propel the center of mass dominates. 
In single support, we found that after scaling for size and speed, $\hat{k}$ is approximately $40 \%$ lower in our child group, they did modulate $\hat{k}$ in the same way as adults. As shown in Fig. 4B, both young children and adults increased $\hat{k}$ when meeting the mechanical demands of walking at fixed speed, but at relatively slower and faster step frequencies. The tendency of children to modulate $\hat{k}$ was independent of changes in the amount of positive work generated by their trailing limb during double support. This behavior deviated from that observed in adults, where the positive work generated by the trailing limb during double support decreased and $\hat{k}$ increased across slow to fast step frequencies. Unlike trailing limb work, which has been shown to rely primarily on plantar flexor muscles and tendons (Fukunaga et al., 2001; Ishikawa et al., 2005; Sawicki and Khan, 2016), $\hat{k}$ can be influenced by multiple muscles and joints, such as the ankle, knee, and hip. Thus, it is possible that both the more compliant $\hat{k}$ and inability to generate as much scaled work and power by the trailing limb may be related to a young child's developing muscular capacity. Over time, as children learn to coordinate their leg and hip muscles to generate propulsive work, the motion of the center of mass during walking may become smoother, reflecting patterns that are observed in adults. Given evidence of structural differences of immature plantar flexor muscle and tendon (Radnor et al., 2018; Waugh et al., 2012) and functional differences in muscle and tendon interaction in children measured directly by ultrasound while hopping (Waugh et al., 2017), it seems likely that the coordination of the plantar flexor muscle-tendon mechanics responsible for efficient propulsion and redirection of the center of mass in adults is not fully formed and learned at 5-6 years of age.

The unequal contribution of work by the trailing limb in young children could also help explain their decreased efficiency (Schepens et al 2004), since positive work is necessary to restore the energy that is lost from the unavoidable collision phase of double support (Donelan et al., 2002). Lost energy must then be replaced with more costly compensation strategies, possibly at other joints such as the hip (Lye et al., 2016; Sawicki et al., 2009), and with coactivation of

422 we observed in our 5-6-year-old child group. Consistent with previous studies (DeJaeger et al., 2001; Morgan et al., 2002), our children had a higher net COT at each step frequency condition, 
even after accounting for differences in Froude speed. When compared to adults, however, the net COT in children did not fluctuate much in response to changes in step frequency (Figure 4C). Thus, we reject our third hypothesis that when compared to adults, walking at the lowest step frequency condition would be relatively more costly for children.

During the first 7-13 years of life, walking patterns vary in their maturity, so interpretation of our results is limited to children aged 5-6 years. At this age, we observed a high amount of variability in the ground reaction force patterns, consistent with previous studies (Kraan et al., 2017). This variability ultimately meant that we were only able to reliably take 30 steps from each trial that met our condition of a reasonable linear fit between force and spring length changes during single support. It is possible that the variability in ground reaction force patterns might have another purpose, such as for motor learning, where the goal is to learn how to efficiently redirect the center of mass. Indeed, the complex relationships between age, size, mechanics, and the spatiotemporal components of walking remain to be understood, and we lack a complete understanding of their contributions to the higher net COT observed in children. Our results should be interpreted with caution because the sample size was small, though it is worth noting that our effect sizes (Cohen's $d$ ) are between 1.07-10 (Table 2), which may be helpful for future reference.

In summary, when compared to adults, we found that when walking at a fixed speed, but at relatively slow and fast step frequencies, 5-6-year-old children generated significantly less positive work by their trailing limb during double support. We also found that $\hat{k}$, the spring-like behavior of the leg, was much more complaint in children than adults. These variables were scaled to speed, mass, and leg length, yet the mechanics of walking in children departed substantially from adults, who have long been seen as the ideal behavior for economical walking. Altogether, our findings suggest that the simple models used here cannot be scaled down and used to adequately characterize a child's walking mechanics and energetics. The implications from this work are worth further consideration because simple models form the basis for more complex theoretical and computational models and aid in the design of assistive devices such as lower limb prostheses and orthoses (Delp et al., 2007; Gard and Childress, 2001; Geyer and Herr, 2010). As opposed to relying on models that are scaled down versions of adults, we propose that a limb-level biomechanical analysis of walking in children may facilitate the 
454 selection and tuning of assistive devices that are specifically designed for children. It is well 455 known that adults can successfully tune and utilize the Achilles tendon to store and release 456 energy to generate propulsive work (Fukunaga et al., 2001; Ishikawa et al., 2005; Lichtwark and 457 Wilson, 2008), directing the attention of engineers and clinicians to the ankle as a first line target 458 for assistive technology in gait rehabilitation. However, little is understood about plantar flexor 459 muscle-tendon function in children, who also require this technology and, in our study, did not 460 generate comparable positive propulsive work by their trailing limb. Combining a limb-level 461 biomechanical analyses with ultrasound measurements of plantar flexor muscle-tendon function 462 is of future interest, as this would help understand the unique strategies that children use to meet 463 the mechanical power demands of walking across a range of speeds and uphill/downhill 464 conditions. 


\section{Acknowledgements}

467 We thank the children and their parents for their participation in this study. We acknowledge 468 Anna Larsson, Daisey Vega, and Danny Guevara for their assistance with data collection and for

469 UH department of Health and Human Performance for supporting the completion of this study 470 and manuscript preparation.

471

472 Competing interests

473 No competing interests declared.

474

475 Author Contributions

476 Conceptualization: V.L.R., C.J.A.; Methodology: V.L.R., C.J.A.; Software: V.L.R., C.J.A.; 477 Validation: V.L.R., C.J.A.; Formal analysis: V.L.R., C.J.A.; Investigation: V.L.R; Resources: 478 C.J.A.; Data curation: V.L.R., C.J.A.; Writing - original draft: V.L.R; Writing - review \& 479 editing: V.L.R., C.J.A.; Visualization: V.L.R, C.J.A.; Supervision: C.J.A.; Project 480 administration: C.J.A.; Funding acquisition: C.J.A.

$483 \quad$ Funding

484 This work was supported by faculty funds provided to C.J.A. 


\section{References}

487

488

489

490

491

492

493

494

495

496

497

498

499

500

501

502

503

504

505

506

507

508

509

510

511

512

513

514

515

516

517

Adamczyk, P. G. and Kuo, A. D. (2009). Redirection of center-of-mass velocity during the step-to-step transition of human walking. J Exp Biol 212, 2668-2678.

Alexander, R. M. (1989). Optimization and gaits in the locomotion of vertebrates. Physiological Reviews.

Antoniak, G., Biswas, T., Cortes, N., Sikdar, S., Chun, C. and Bhandawat, V. (2019). Spring-loaded inverted pendulum goes through two contraction-extension cycles during the single-support phase of walking. Biol Open $\mathbf{8}$,

Arellano, C. J., O'Connor, D. P., Layne, C. and Kurz, M. J. (2009). The independent effect of added mass on the stability of the sagittal plane leg kinematics during steady-state human walking. Journal of Experimental Biology 212, 1965-1970.

Blickhan, R. (1989). The spring-mass model for running and hopping. Journal of Biomechanics 22, 1217-1227.

Bolster, E. A. M., Balemans, A. C. J., Brehm, M.-A., Buizer, A. I. and Dallmeijer, A. J. (2017). Energy cost during walking in association with age and body height in children and young adults with cerebral palsy. Gait \& Posture 54, 119-126.

Brenière, Y. and Bril, B. (1998). Development of postural control of gravity forces in children during the first 5 years of walking. Experimental Brain Research 121, 255-262.

Bril, B., Dupuy, L., Dietrich, G. and Corbetta, D. (2015). Learning to tune the antero-posterior propulsive forces during walking: a necessary skill for mastering upright locomotion in toddlers. Exp Brain Res 233, 2903-2912.

Brockway, J. M. (1987). Derivation of Formulae Used To Calculate Energy Expenditure in Man. Human Nutrition: Clinical Nutrition 41C, 463-471.

Cavagna, GA and Margaria, R (1966). Mechanics of Walking. Journal of Applied Physiology 21, 271-278.

Chester, V. L., Tingley, M. and Biden, E. N. (2006). A comparison of kinetic gait parameters for 3-13 year olds. Clinical Biomechanics 21, 726-732.

DeJaeger, D., Willems, P. A. and Heglund, N. C. (2001). The energy cost of walking in children. Pflugers Archiv European Journal of Physiology 441, 538-543.

Dewolf, A. H., Sylos-Labini, F., Cappellini, G., Lacquaniti, F. and Ivanenko, Y. (2020). Emergence of Different Gaits in Infancy: Relationship Between Developing Neural Circuitries and Changing Biomechanics. Front. Bioeng. Biotechnol. 8,. 
Dierick, F., Lefebvre, C., Van Den Hecke, A. and Detrembleur, C. (2004). Development of displacement of centre of mass during independent walking in children. Developmental Medicine \& Child Neurology 46, 533-539.

Donelan, J. M., Kram, R. and Kuo, A. D. (2002). Mechanical work for step-to-step transitions is a major determinant of the metabolic cost of human walking. Journal of Experimental Biology 205, 3717-3727.

Enoka, R. M. (2015). Neuromechanics of human movement. Fifth edition. Champaign, IL: Human Kinetics.

Fukunaga, T., Kubo, K., Kawakami, Y., Fukashiro, S., Kanehisa, H. and Maganaris, C. N. (2001). In vivo behaviour of human muscle tendon during walking. Proc Biol Sci 268, 229-233.

Geyer, H., Seyfarth, A. and Blickhan, R. (2006). Compliant leg behaviour explains basic dynamics of walking and running. Proceedings of the Royal Society B: Biological Sciences 273, 2861-2867.

Glass, G. V. and Hopkins, K. D. (2008). Statistical Methods in Education and Psychology. 3rd ed. University of Colorado, Boulder: Pearson College Div.

Grabowski, A., Farley, C. T. and Kram, R. (2005). Independent metabolic costs of supporting body weight and accelerating body mass during walking. Journal of Applied Physiology 98, 579-583.

Grosset, J. F., Mora, I., Lambertz, D. and Pérot, C. (2008). Voluntary activation of the triceps surae in prepubertal children. Journal of Electromyography and Kinesiology 18, 455465.

Hallemans, A., Aerts, P., Otten, B., Deyn, P. P. D. and Clercq, D. D. (2004). Mechanical energy in toddler gait A trade-off between economy and stability? Journal of Experimental Biology 207, 2417-2431.

Heglund, N. C. and Taylor, C. R. (1988). Speed, stride frequency and energy cost per stride: how do they change with body size and gait? Journal of Experimental Biology 138, 301318.

Ishikawa, M., Komi, P. V., Grey, M. J., Lepola, V. and Bruggemann, G.-P. (2005). Muscletendon interaction and elastic energy usage in human walking. Journal of Applied Physiology 99, 603-608.

Kraan, C. M., Tan, A. H. J. and Cornish, K. M. (2017). The developmental dynamics of gait maturation with a focus on spatiotemporal measures. Gait \& Posture 51, 208-217.

Kramer, P. A. and Sarton-Miller, I. (2008). The energetics of human walking: Is Froude number (Fr) useful for metabolic comparisons? Gait \& Posture 27, 209-215. 
Kramer, P. A. and Sylvester, A. D. (2013). Humans, geometric similarity and the Froude number: is "reasonably close" really close enough? Biology Open 2, 111-120.

Kuo, A. D., Donelan, J. M. and Ruina, A. (2005). Energetic Consequences of Walking Like an Inverted Pendulum: Step-to-Step Transitions: Exercise and Sport Sciences Reviews 33, 88-97.

Lambertz, D., Mora, I., Grosset, J.-F. and Pérot, C. (2003). Evaluation of musculotendinous stiffness in prepubertal children and adults, taking into account muscle activity. Journal of Applied Physiology 95, 64-72.

Lichtwark, G. A. and Wilson, A. M. (2008). Optimal muscle fascicle length and tendon stiffness for maximising gastrocnemius efficiency during human walking and running. Journal of Theoretical Biology 252, 662-673.

Lye, J., Parkinson, S., Diamond, N., Downs, J. and Morris, S. (2016). Propulsion strategy in the gait of primary school children; the effect of age and speed. Human Movement Science 50, 54-61.

Malloggi, C., Rota, V., Catino, L., Malfitano, C., Scarano, S., Soranna, D., Zambon, A. and Tesio, L. (2019). Three-dimensional path of the body centre of mass during walking in children: an index of neural maturation. International Journal of Rehabilitation Research 42, 112-119.

McMahon, T. A., Valiant, G. and Frederick, E. C. (1985). Groucho Running. Journal of Applied Physiology 62, 2326-37.

Morgan, D. W., Tseh, W., Caputo, J. L., Keefer, D. J., Craig, I. S., Griffith, K. B., Akins, M.-B., Griffith, G. E. and Martin, P. E. (2002). Longitudinal profiles of oxygen uptake during treadmill walking in able-bodied children: the locomotion energy and growth study. Gait \& Posture 15, 230-235.

Preis, S., Klemms, A. and Müller, K. (1997). Gait analysis by measuring ground reaction forces in children: changes to an adaptive gait pattern between the ages of one and five years. Developmental Medicine \& Child Neurology 39, 228-233.

Radnor, J. M., Oliver, J. L., Waugh, C. M., Myer, G. D., Moore, I. S. and Lloyd, R. S. (2018). The Influence of Growth and Maturation on Stretch-Shortening Cycle Function in Youth. Sports Med 48, 57-71.

R.M. Alexander and A.S. Jayes (1983). A dynamic similarity hypothesis for the gaits of quadrupedal animals. J.Zool. 201, 135-152.

Sawicki, G. S. and Khan, N. S. (2016). A Simple Model to Estimate Plantarflexor MuscleTendon Mechanics and Energetics During Walking With Elastic Ankle Exoskeletons. IEEE Trans Biomed Eng 63, 914-923. 
Sawicki, G. S., Lewis, C. L. and Ferris, D. P. (2009). It Pays to Have a Spring in Your Step: Exercise and Sport Sciences Reviews 37, 130-138.

Schepens, B. (2004). Mechanical work and muscular efficiency in walking children. Journal of Experimental Biology 207, 587-596.

Takegami, M.D., Y. (1992). Wave Pattern of Ground Reaction Force of Growing Children. Journal of Pediatric Orthopaedics 12, 522-526.

Usherwood, J. R., Hubel, T. Y., Smith, B. J. H., Self Davies, Z. T. and Sobota, G. (2018a). The scaling or ontogeny of human gait kinetics and walk-run transition: The implications of work vs. peak power minimization. Journal of Biomechanics 81, 12-21.

Usherwood, J. R., Hubel, T. Y., Smith, B. J. H., Davies, Z. S. and Sobota, G. (2018b). The scaling or ontogeny of human gait kinetics and walk-run transition: The implications of work vs. peak power minimization. Journal of Biomechanics; Kidlington 81, 12-21.

Waugh, C. M., Blazevich, A. J., Fath, F. and Korff, T. (2012). Age-related changes in mechanical properties of the Achilles tendon. Journal of Anatomy 220, 144-155.

Waugh, C., Korff, T., Fath, F. and Blazevich, A. (2013). Rapid Force Production in Children and Adults: Mechanical and Neural Contributions. Medicine \& Science in Sports \& Exercise 45, 762-771.

Waugh, C. M., Korff, T. and Blazevich, A. J. (2017). Developmental differences in dynamic muscle-tendon behaviour: implications for movement efficiency. The Journal of Experimental Biology 220, 1287-1294. 


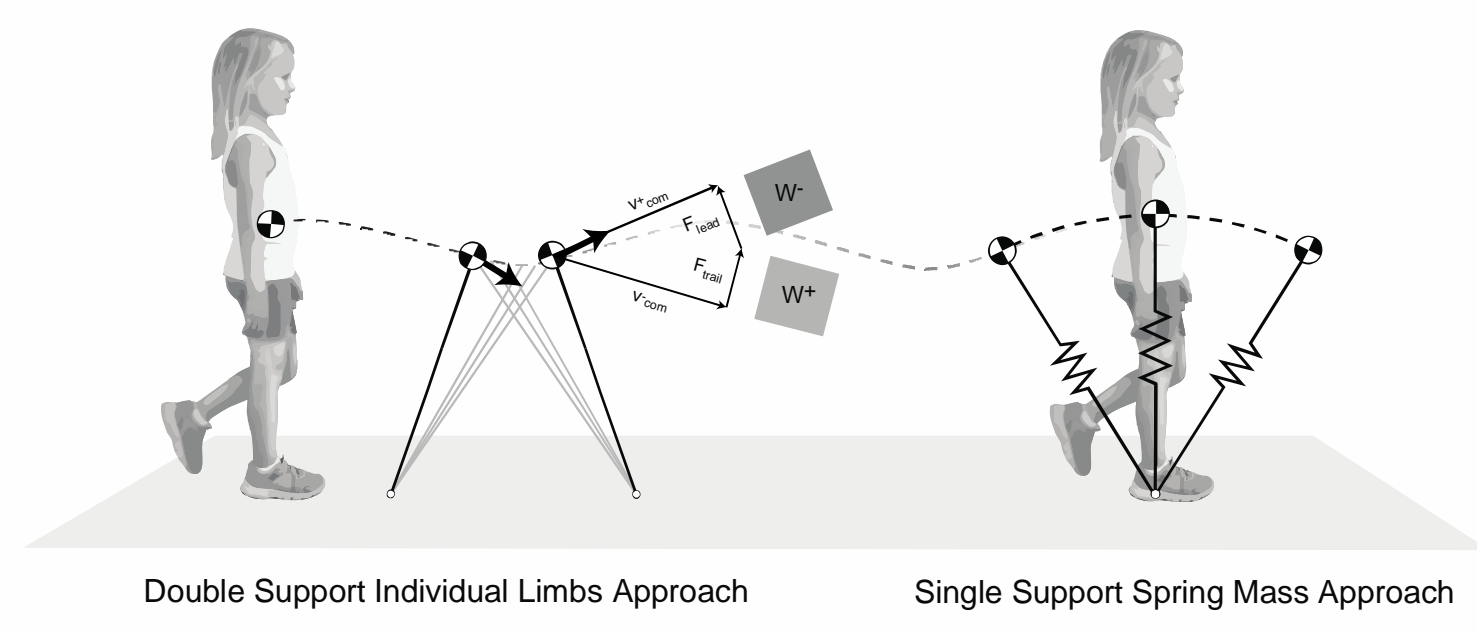

Figure 1. Geometric diagrams illustrate the templates used to analyze the mechanics of walking in children. For both templates, ground reaction force data were used to calculate center of mass velocity and position. Following the dynamic walking model, (left) we quantified the mechanical work generated by the individual limbs during double support (Donelan et al. 2002), a key determinant in transitioning the body's center of mass from the trailing leg to the lead leg. In recognizing the contributions of elastic energy storage and return to the work done on the center of mass, we quantified the spring stiffness, $k$, of the leg as proposed by the spring-mass model (Geyer et al). As a simple approach, we quantified the spring-like behavior of the leg during single support, as this is the period when the leg "spring" would undergo energy release as the spring extends and the center of mass reaches it maximum height at midstance. Then, as the center of mass moves forward and its height decreases, the spring compresses and stores energy. The diagram depicting the step-to-step transition during double support is modified after Kuo et al. (2005). 


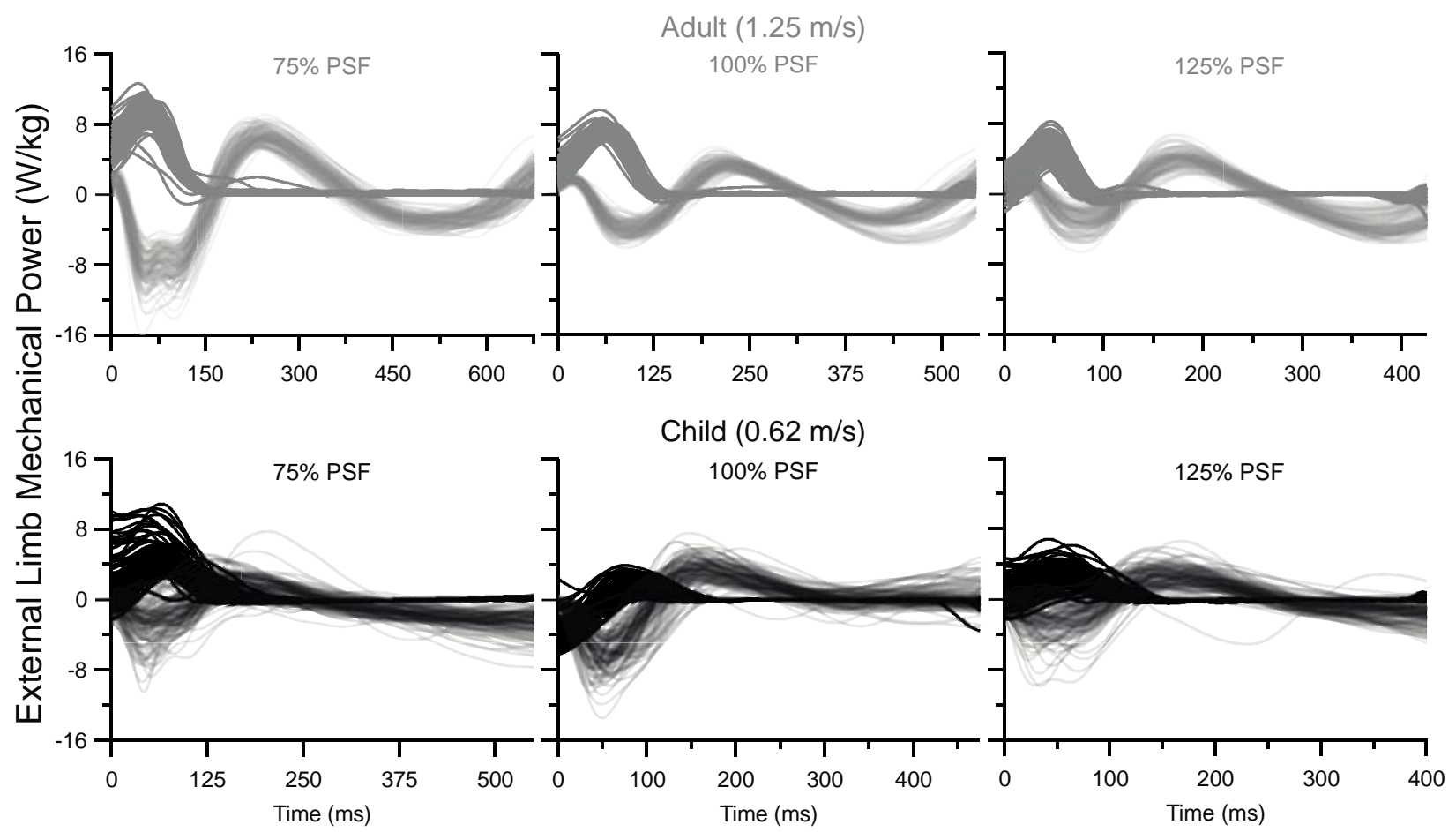

638

639

640

641

642

643

644

645

646

647

648

649

650

651

652

653

Figure 2. Examples of an adult (gray lines, top) and child (black lines, bottom) external mechanical power generated by the trailing (dark) and leading (light) leg during consecutive steps, under conditions of walking at their self-selected speed at $75 \%, 100 \%$, and $125 \%$ of preferred step frequency (PSF). When walking at $1.25 \mathrm{~m} / \mathrm{s}$ and at $100 \%$ PSF, the external mechanical power generated by the legs follows the typical pattern observed in young, healthy adults (Donelan et al. 2002), with the trailing and leading leg generating roughly equal positive and negative power during double support. In contrast, when walking at their self-selected speed of $0.62 \mathrm{~m} / \mathrm{s}$ at $100 \%$ PSF, the child's leading and trailing legs transitioned from generating negative to positive external mechanical power during double support. In general, when walking at a fixed speed, but at variable step frequencies, the adult modulated the magnitude of the positive external mechanical power generated by their trailing leg, with the slower step frequency coinciding with higher positive power and the faster step frequency coinciding with lower negative power. The child, however, exhibited a strategy whereby the trailing leg no longer generates negative and positive power during double support (as observed at 100\% PSF), but primarily generates positive power when walking at the slower and faster step frequency. To highlight the greater amount of variability observed in our child group, plots of external mechanical power as a function of time consist of 100 consecutive steps. 
A

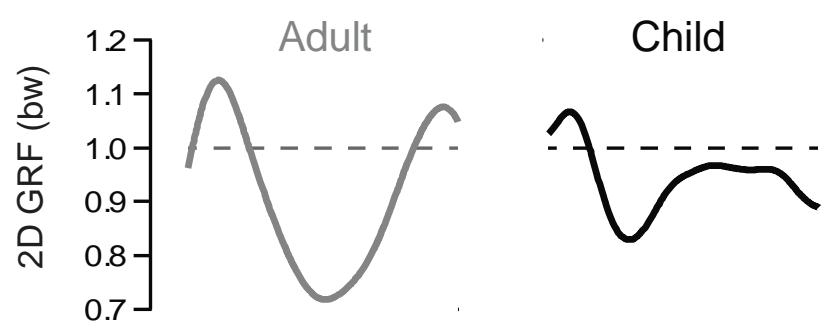

B

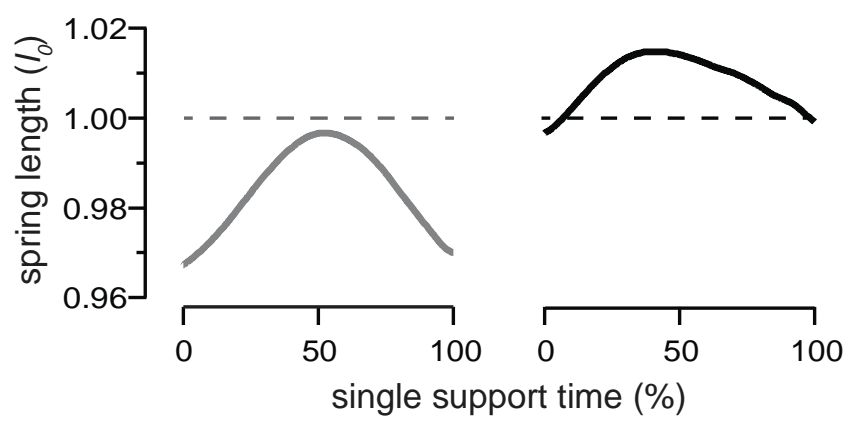

C

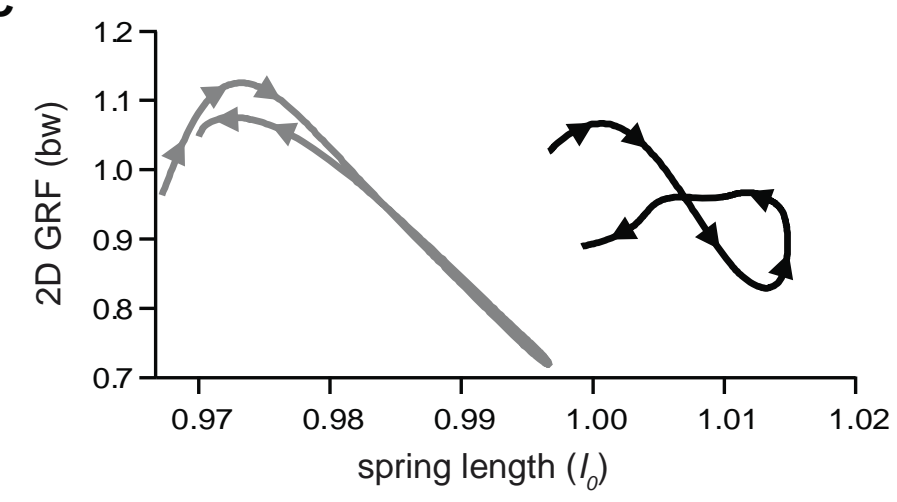

Figure 3. Representative adult (left, gray) and child (right, black) force and spring length curves during single support phase at preferred step frequency. (A) 2D resultant GRF scaled to bodyweight (bw) and (B) 2D spring length as measured from the COM to centre of pressure, scaled to standing height of COM $\left(l_{0}\right)(\mathbf{C})$ Force-length curves show greater asymmetry and a flatter slope for a child subject. Positive mechanical work at rebound (early single support) reflects recoil of the leg spring just after collision, while negative mechanical work in preload (late single support) reflects compression of the leg spring in preparation for propulsion. The convention here is that the absolute value of the slope equals the normalized spring stiffness, $\square$. 


\section{A}
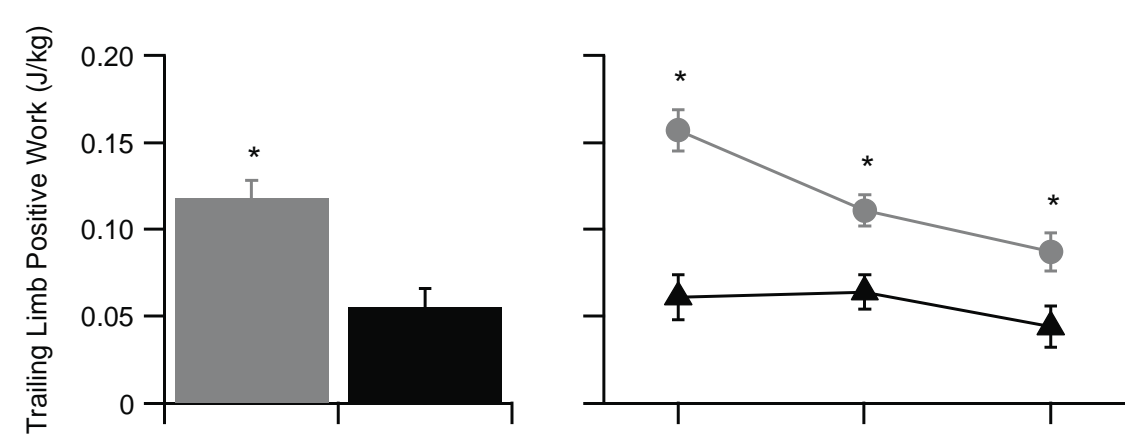

\section{B}
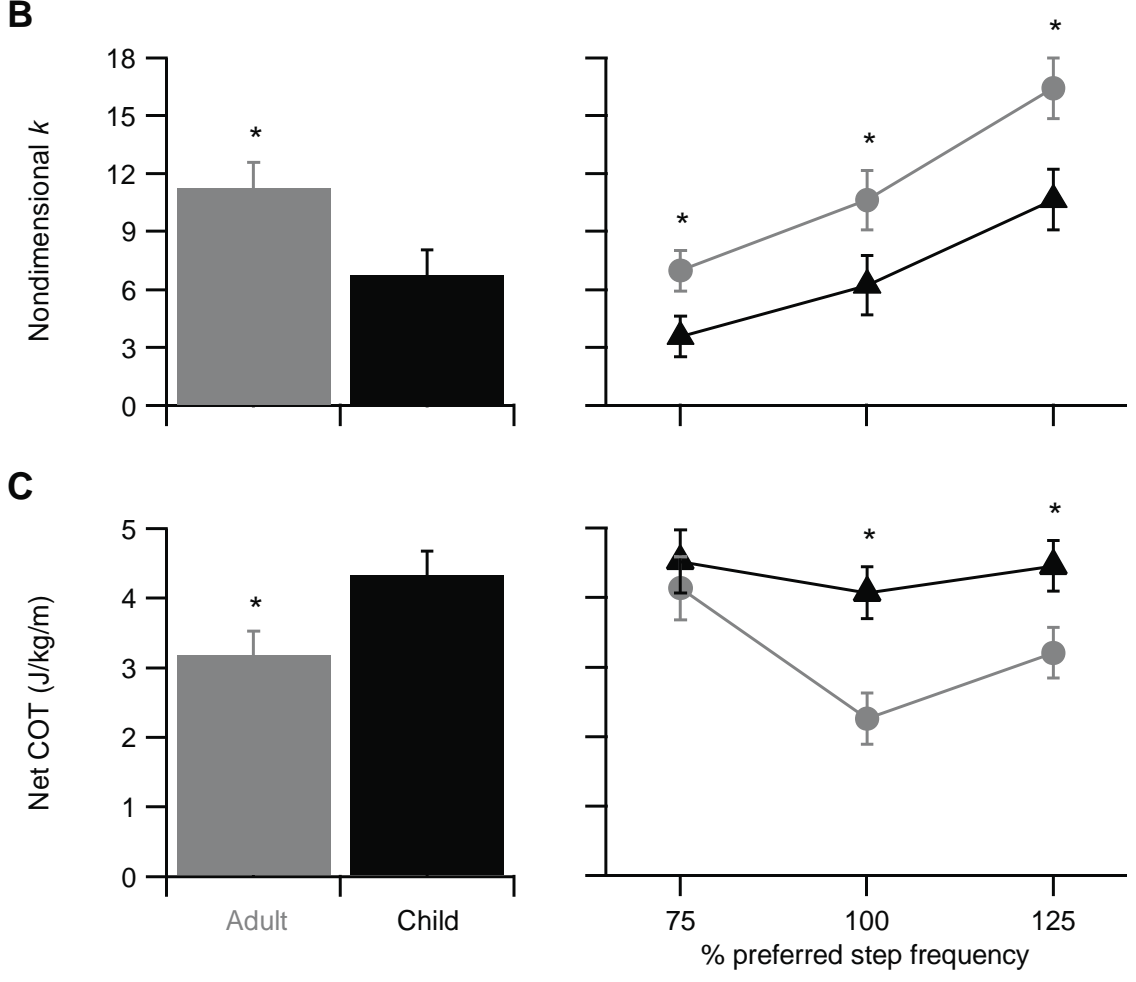

Figure 4. (A) The positive work generated by the trailing limb was smaller in children as compared to adults (left; *significant group effect $p=0.039$ ). In response to walking at relatively slow, preferred, and fast strep frequencies, adults (grey) altered the positive work generated by the trailing limb, however, children (black) showed little to no change in trail limb positive work (right; *significant interaction effect $p=0.013$ ). (B) When compared to adults, children operated their leg stiffness with a lower $\hat{k}$ (left; *significant group effect $p<0.001$ ); however, when walking across a range of relatively slow and fast step frequencies, both children and adults modulated $\hat{k}$ in the same way (right; no interaction effect $p$ $=0.41$ ). (C) Differences in trailing limb positive work and $\hat{k}$ were associated with differences in the net COT required to walk. Overall, children walked with a $36 \%$ higher net COT (left; *significant group effect $p=0.03$ ), yet, both children and adults increased their net COT in response to walking at relatively slow and fast step frequencies, exhibiting a U-shaped trend (right; no interaction effect $p>.05$ ). Note that for the repeated measures ANCOVAs, average values for trail limb positive work and net COT are adjusted at a Froude number equal to 0.3. For all values, the bars represent the standard error of the mean. Significant differences between children and adults at each step frequency (right) are marked by an *, 
A

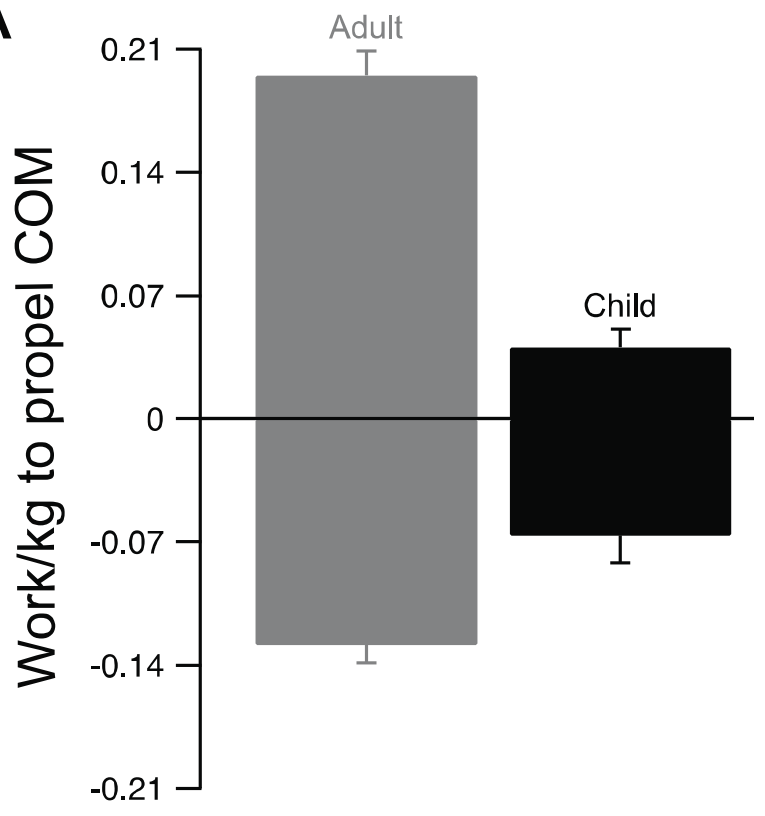

B

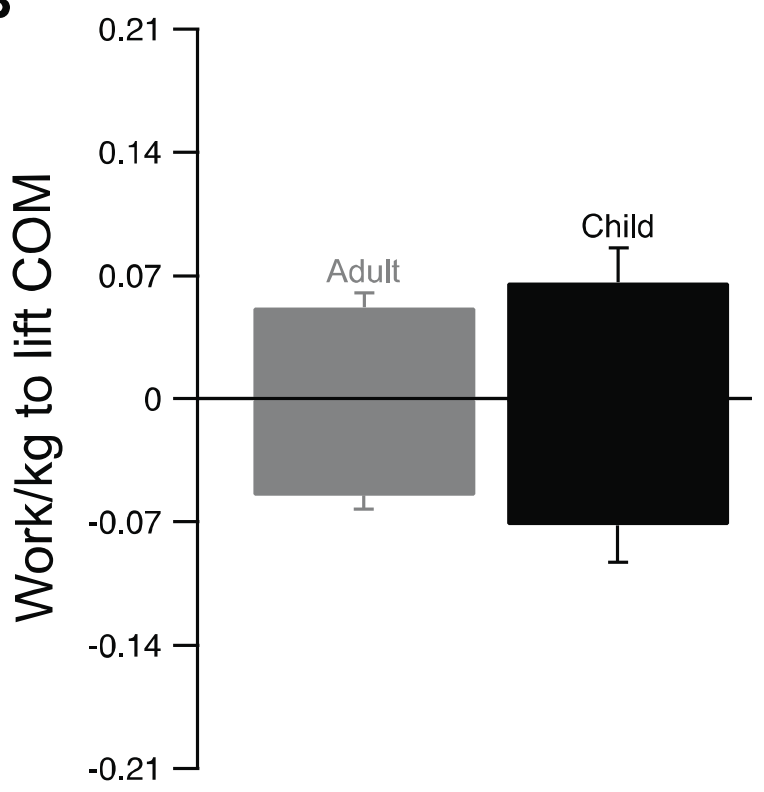

Figure 5. Partitioning the mean mass-specific work of both limbs reveal that children performed (A) much less work to propel the COM than adults, but (B) slightly higher work to lift the COM. As noted in the textNote that the trailing limb in the child group performed both positive and negative work in double support, while the trail limb in the adult group was only used to generate positive work. 
Table 1: Subject Characteristics

\begin{tabular}{lll}
\hline & Children & Adults \\
\hline Sex $(\mathrm{M} / \mathrm{F})$ & $4 / 4$ & $4 / 4$ \\
Age $(\mathrm{y})$ & $5.43 \pm 0.53$ & $26.38 \pm 4.40$ \\
Height (m) & $1.22 \pm 0.04$ & $1.72 \pm 0.08$ \\
Mass (kg) & $24.77 \pm 5.60$ & $75 \pm 17.40$ \\
Spring length (m) & $0.70 \pm 0.06$ & $1.03 \pm 0.06$ \\
Leg length (m) & $0.62 \pm 0.03$ & $0.92 \pm 0.05$ \\
Preferred speed (m/s) & $0.52 \pm 0.13$ & $1.13 \pm 0.18$ \\
Froude number & $0.20 \pm 0.01$ & $0.40 \pm 0.01$ \\
Step frequency achieved vs. & $2.47 \pm 2.73$ & $0.66 \pm 1.30$ \\
cued (\% difference) & & \\
\hline \multicolumn{2}{l}{ Values are mean \pm SD, Percent difference in step frequency is at the } \\
preferred frequency condition & &
\end{tabular}


Table 2. Descriptive Statistics, Pairwise and Planned Comparisons

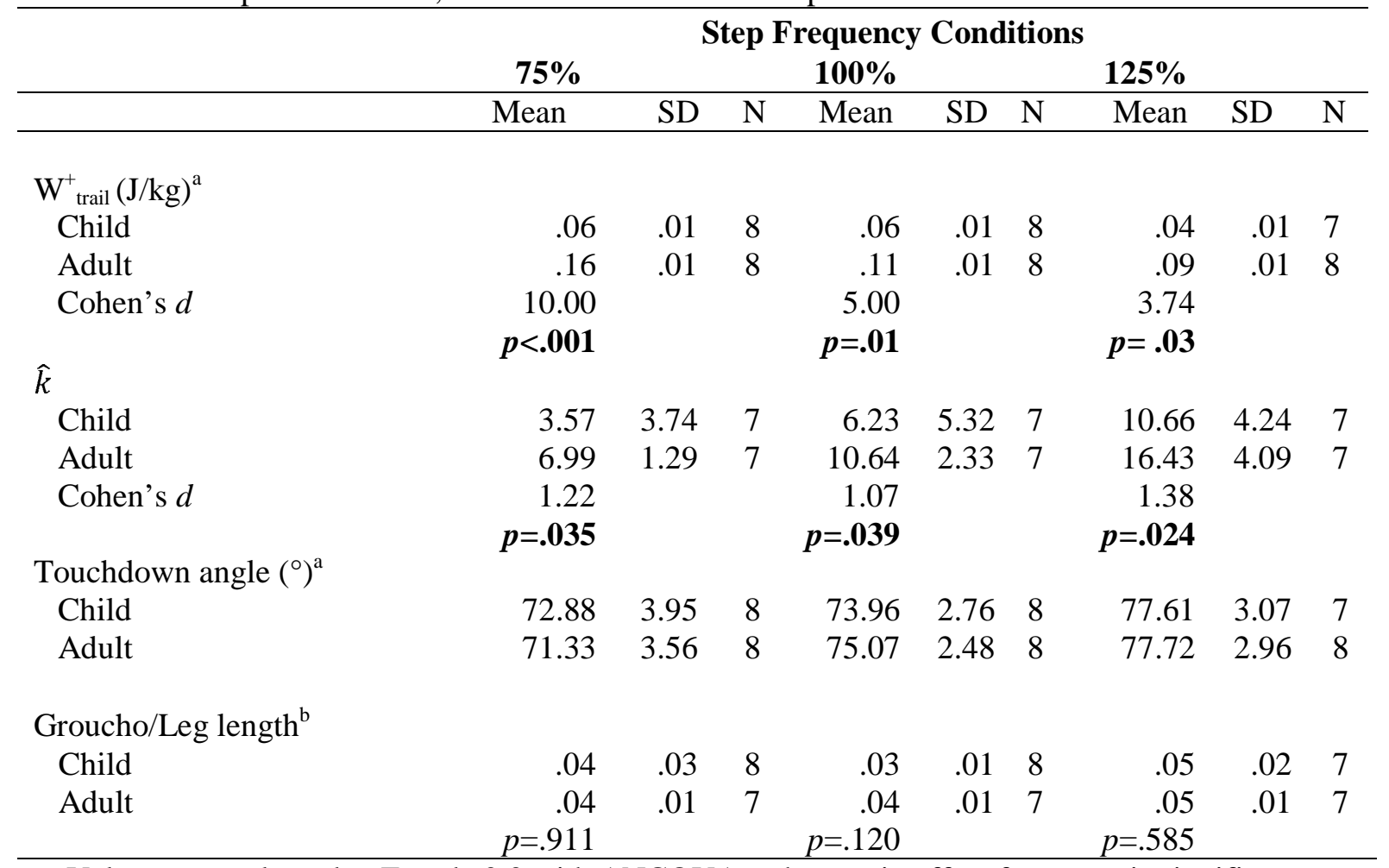

a. Values are evaluated at Froude 0.3 with ANCOVAs when main effect for group is significant; comparisons with $\square$ were followed up with a priori $t$-test when significant. b. Independent $t$-test 
Table 3 Metabolic R-ANCOVA results, and ANCOVA pairwise comparisons

$\begin{array}{lll}\text { Effects }^{a} & \boldsymbol{F} & p\end{array}$

Net Cost of Transport $(\mathrm{J} / \mathrm{kg} / \mathrm{m})$

\begin{tabular}{|c|c|c|}
\hline Step frequency & $F=3.805, d f=2,26$ & $0.036 *$ \\
\hline Group & $F=4.003, d f=1,13$ & $0.034 *$ \\
\hline Group x Step frequency & $F=2.112, d f=2,26$ & 0.071 \\
\hline \multicolumn{3}{|l|}{ Conditions } \\
\hline Children and Adults at $75 \%$ & $\mathrm{~F}=0.232, \mathrm{df}=1,16$ & 0.319 \\
\hline \multirow[t]{2}{*}{ Children and Adults at $100 \%{ }^{b}$} & $F=7.653, d f=1,16$ & $0.008 * *$ \\
\hline & $U=49$ & $0.001 * *$ \\
\hline Children and Adults at $125 \%$ & $F=3.752, d f=1,16$ & 0.038* \\
\hline
\end{tabular}

a. Mean differences evaluated at Froude $0.3 ; \mathbf{b}$. Comparison violated assumption of homogeneity of variances, so differences analyzed with a Mann-Whitney $\mathrm{U}$ test; ${ }^{*} p<.05,{ }^{*} p<.01$ 